\title{
La sauvegarde des intérêts piscicoles au cours des travaux d'aménagement des cours d'eau
}

\author{
PAR \\ J. Arrignon \\ Ingénieur IAN \\ Chargé de la Région piscicole de Compiègne \\ au Conseil supérieur de la Pêche \\ G. Rouyer \\ Ingénieur en Chef \\ du Génie rural des Eaux et des Forêts \\ Chargé de la Région piscicole de poitier \\ au Conseil supérieur de la Pêche \\ ET \\ F. Chancerel \\ Ingénieur ENSAR \\ Région piscicole de Poitiers
}

\section{1 - Introduction}

\section{1 - SITUATION}

Les cours d'eau sont les vecteurs naturels des eaux superficielles et parfois d'une part des ressources souterraines. Les activités humaines font que leur situation et leur état relativement stationnaires et en rapport normal avec leur vocation par le passé (moyennant de nécessaires travaux d'entretien) subissent présentement une évolution dont la rapidité est croissante et dont les effets sont nuisibles:

- à la vocation de vecteur physique d'un élément naturel : l'eau;

- à la vocation du milieu biologique, piscicole que constitue le vecteur.

Les aménagements hydrauliques des cours d'eau ont pour but de combattre les effets nuisibles à la vocation de vecteur. Ils combattent parfois les effets nuisibles à la vocation de milieu biologique, mais ils perturbent également et souvent la vocation elle-même de milieu biologique.

\section{2 - APPROCHE DU PROBLËME}

La sauvegarde des intérêts piscicoles doit donc être étudiée et intégrée dans les aménagements hydrauliques des cours d'eau. Il convient de passer en revue les grands types d'aménagement en définissant leurs objectifs, la façon dont ils sont conçus et réalisés et leur incidence piscicole. Ces grands types se répartissent en deux groupes selon l'objectif poursuivi :
1.2.1 - Les aménagements ayant pour but de faciliter l'écoulement des eaux en vue de :

- supprimer l'inondation des zones habitées ou des voies de communication;

- assainir les zones plus ou moins marécageuses;

- réduire le temps de submersion des terrains du lit majeur;

en distinguant les travaux d'entretien et les travaux neufs.

1.2.2 - Les aménagements ayant pour but le stockage en vue de :

- l'utilisation de l'eau dont on ne retiendra que les écluses et barrages des usines «au fil de l'eau», les lacs artificiels et retenues collinaires entrant dans un ordre de préoccupations différent.

\section{2 - Les aménagements hydrauliques}

\section{1 - LES AMÉNAgEMENTS AYANT POUR BUT} DE FACILITER L'ÉCOULEMENT DES EAUX

\subsection{1 - Les travaux d'entretien}

\subsubsection{1 - Situation actuelle.}

L'entretien du cours d'eau est toujours à la charge du propriétaire riverain, qu'il s'agisse du particulier ou de l'Etat.

Alors que, par le passé, le particulier avait les moyens physiques et financiers d'opérer ou de faire opérer régulièrement l'entretien des berges, le faucardement et le curage, 
il n'en est plus de même présentement. L'évolution du monde agricole entrave le «jardinage des rivières »: la main-d'œuvre est chère sinon rare, l'émondage et le bûcheronnage des rives boisées n'apportent aucun profit: on se chauffe au fuel et non plus au bois. Le remodelage du paysage rural accélère l'érosion et la sédimentation. L'activité du rat musqué (Ondatra) aggrave l'état de berges souvent bouleversées par le piétinement d'un cheptel en surnombre dans les prairies riveraines (fig. 1).

Pour ce qui concerne le domaine public, les cours d'eau sont régulièrement entretenus tant qu'ils présentent un intérêt pour la navigation. Le déclassement de partie d'entre eux est le départ de leur dégradation. $\mathrm{Si}$, à court terme, l'amélioration de leurs caractéristiques piscicoles est réelle, le défaut d'entretien des ouvrages, des rives et des berges transforme les tronçons déclassés en eaux stagnantes, rapidement eutrophes puis séniles au plan piscicole (Seine, Bras de Guernes, Yvelines, fig. 2).

L'intervention des moyens mécaniques est possible mais onéreuse pour un travail apparemment peu productif. Les difficultés de financement font qu'ils sont rares ou qu'ils sont introduits dans le cadre d'interventions subventionnées par l'Etat et par les Collectivités : recalibrages, accompagnés assez souvent de reprofilage, de rectifications du lit.

Il en résulte des actions intempestives et anarchiques en raison du manque de coordination au niveau des études, du financement, de la décision et de l'exécution : les travaux sont ouverts à n'importe quelle époque de l'année, en fonction, par exemple de crédits disponibles ou des désidérata les plus divers des riverains.

Il en résulte, a contrario, une absence totale d'entretien due à un défaut de financement collectif, à la négligence ou à l'indifférence des propriétaires riverains, à une législation et à une réglementation peu dissuasives.

\subsubsection{2. - CONSÉQUENCES PISCICOLES.}

Les interventions excessives ou intempestives entraînent ordinairement :

- La destruction totale d'un certain nombre de maillons du cycle hydrobiologique: c'est le cas du curage et du faucardement intégral en période estivale. A un fond vaseux, trop organique, est substitué un fond minéral trop stérile. Les organismes décomposeurs ont totalement disparu, de même que les herbiers aquatiques support du periphyton entrant dans la chaîne alimentaire, pâturage des invertébrés et des poissons herbivores, abris de ces derniers et des autres, générateur par photosynthèse de l'oxygène certes nécessaire à l'ensemble de la faune aquatique mais également au processus naturel d'auto-épuration des eaux véhiculées (fig. 3).

- La destruction directe ou indirecte d'une espèce piscicole est encore le fait du curage et du faucardement, même partiels. Un curage hivernal, en amont d'une frayère à salmonidés, entraîne le colmatage de la gravière hébergeant les œufs et l'asphyxie de ces derniers. Un curage estival a le même effet sur les pontes de cyprinidés. Les faucardements précoces entrainent une destruction importante d'alevins de salmonidés qui, après leur émergence des gravières, trouvent abri dans les herbiers.

$\mathrm{Au}$ contraire, l'absence d'entretien provoque:

- Une dégradation fondamentale du milieu aquatique: ralentissement du courant d'eau par des obstacles, dépôt

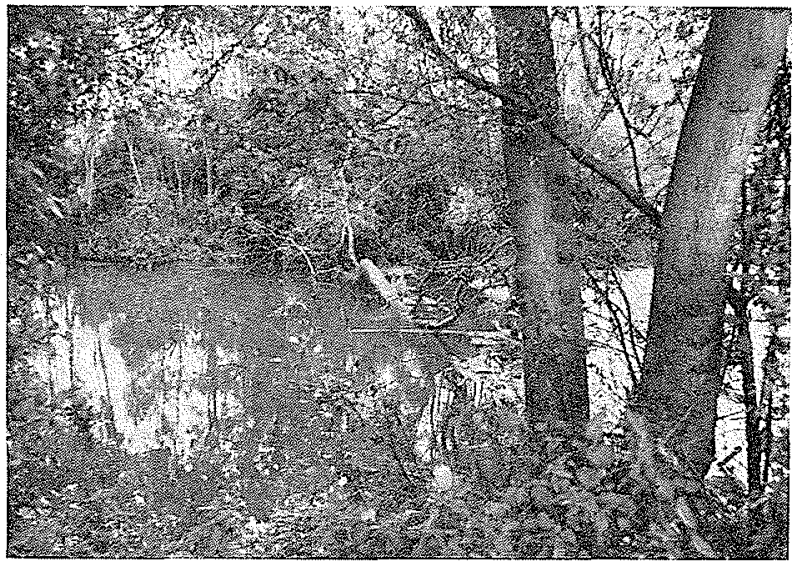

1/ Type de cours d'eau non entretenu - Le lit est encombré, le courant est ralenti, la sédimentation accélérée. Guernes / Seine, Yvelines (J.-J. Demars)

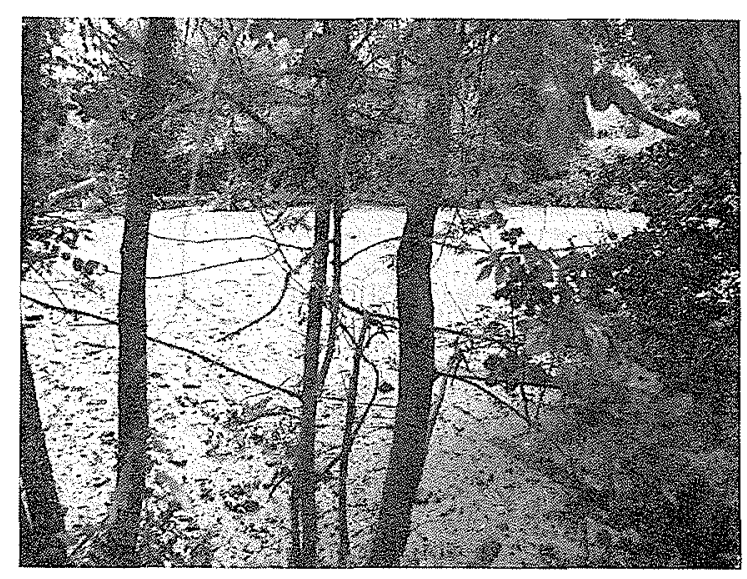

2/ Cours d'eau déclassé, non entretenu - Le courant est nul Le vent dominant accumule les déchets sur une surface recouverte par des lentilles d'eau - Pas de photosynthèse possible sous cet écran.

Bras de Guernes / Seine, Yvelines (J.-J. Demars)

de débris, envasement, fermentation des vases, réchauffement des eaux, diminution de l'éclairement par le développement de surplombs arbustifs ou de voûtes végétales, font que les données physico-chimiques évoluent par exemple d'un caractère lotique à un caractère lentique dégradé.

- L'évolution des données physico-chimiques, notamment en ce qui concerne le ralentissement du courant d'eau, le réchauffement de l'eau, la diminution de la teneur en oxygène et l'accroissement des teneurs en sels ammoniaux, nitrites, nitrates, phosphates et sulfures, entraîne une évolution de la flore et de la faune aquatique. La flore peut disparaitre faute de lumière, le phyto et le zooplancton, le periphyton et le benthos font l'objet de substitutions: les espèces rhéophiles disparaissent, les sténothermes également, qui sont remplacés par des espèces beaucoup plus ubiquistes. L'évolution touche également la population de poissons: directement par le fait que certaines espèces s'accommodent mal de la transformation du milieu physique et chimique.

-- La dégradation des frayères à salmonidés par colmatage, puis leur disparition, touche également le cheptel, 


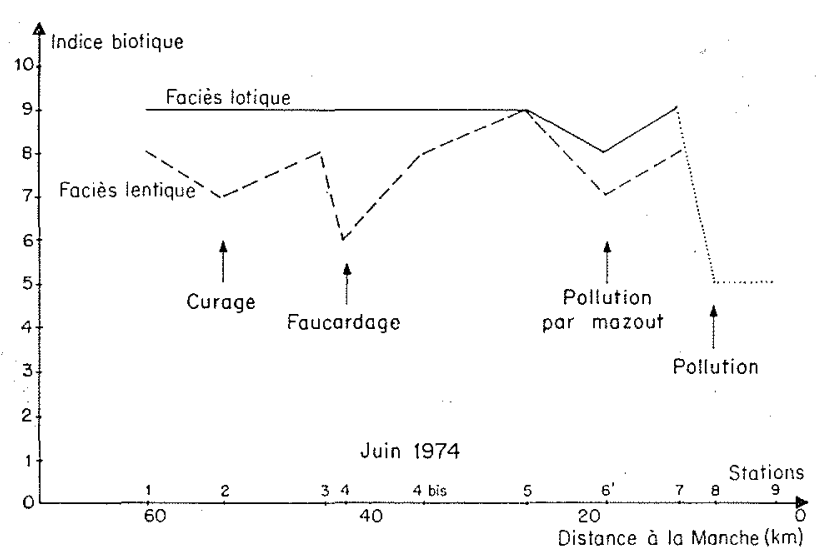

$3 /$ Influence des interventions dans le lit sur les indices biotiques. Bresle/Somme, Seine-Maritime (J.-P. Porcher et C. Porcher).

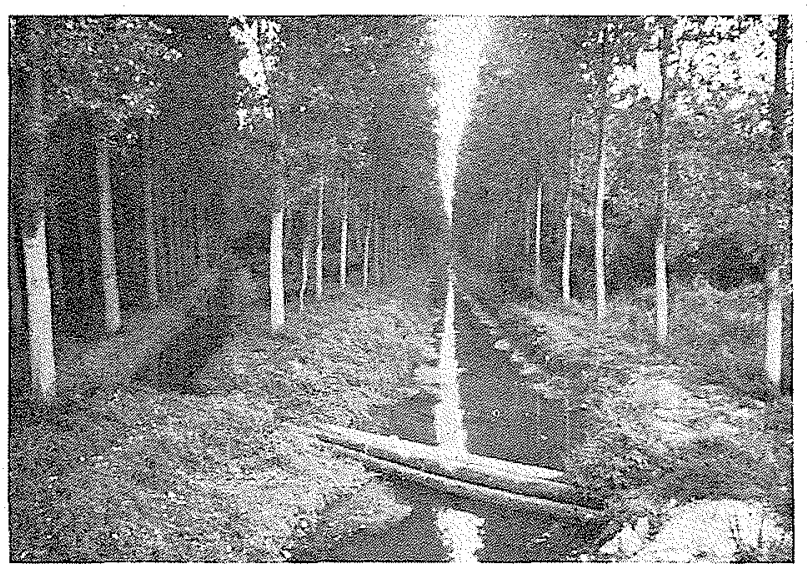

4/ Type de cours d'eau du dọmaine privé, reprofilé et rectiligne - Milieu aquatique sans diversité, sans abri pour les poissons: le fond est nu - Remarquer l'obstacle constifué par les canalisations. Aronde/Oise/Seine, Oise (J. Arrignon).

provoque son vieillissement et, à terme, entraîne un basculement de la dominance: la truite est remplacée par le chevesne, espèce moins exigeante mais économiquement moins intéressante.

- La mancuvre irréfféchie des ouvrages hydrauliques, conjuguée à un défaut d'èntretien, entraîne des dommages allant de la mise en mouvement de dépôts nocifs (sulfures), causant en aval une réelle pollution, à la mise en assec prolongé de frayères à cyprinidés et à brochets, parfois même de piscicultures riveraines (Iton).

\subsection{2 - Les travaux neufs}

\subsubsection{1 - Situation actuelle.}

- Le recalibrage, le reprofilage des cours d'eau, la rectification du lit impliquent la connaissance préalable des débits de crues, de la durée acceptable de submersion des terrains du lit majeur, de la structure des sols traversés.

Dans bien des cas, on ne dispose pas encore d'information sur des débits réels mesurés, soit en raison de l'insuffisance de la couverture en stations de jaugeage, soit en raison du caractère trop récent de leur installation. On est donc amené à déterminer ces débits par le calcul, à partir de données relatives au bassin versant considéré ou par analogie avec un bassin semblable mieux connu.

- Le choix de la durée acceptable de submersion est fonction des intérêts à sauvegarder, suivant qu'il s'agit de zones habitées et de voies de communication, de zones maraîchères, de zones de grande culture, de zones herbagères, de pleupleraies.

Il est également fonction des époques de crues. En zone rurale, le temps de submersion généralement admis résulte d'un compromis fonction des différents types de cultures, ce qui entraîne une grande part de subjectivité : souvent les gabarits sont simplement déterminés pour permettre l'évacuation de la crue décennale. Le manque de moyens immédiats d'étude conduit à la borner à l'aménagement hydraulique du bras principal, à la détermination d'un profil en long à partir de quelques points remarquables, à un nombre de profils en travers souvent trop distants. Cette situation a conduit, par le passé, à l'adoption uniforme de gabarits théoriques, à la création de longues sections rectilignes: la prudence commandait de commettre plutôt des erreurs par excès.

\subsubsection{2 - CONSÉQUENCES.}

- D'un point de vue hydrobiologique et piscicole, la modification des caractéristiques morpho-dynamiques d'un cours d'eau : pente et tracé, géométrie de la section mouillée, vitesse du courant d'eau, entraîne une modification des caractéristiques physico-chimiques et phyto-zoologiques.

Les modifications physico-chimiques se rapportent à un brutal accroissement de l'éclairement, à l'augmentation des écarts thermiques, à l'augmentation des variations de teneurs en $\mathrm{O}_{2}$ et en $\mathrm{CO}_{2}$ dues à la prolifération d'algues génératrices, particulièrement favorisées par l'éclairement et par la minceur de la lame d'eau.

Les modifications phytologiques consistent en une substitution à une végétation aquatique immergée, d'une végétation dressée ou en une prolifération d'algues filamenteuses.

Les modifications de l'habitat tiennent à la disparition des sous-berges, des zones de remous, de mortes et de vives eaux, à la disparition de la végétation immergée et à la stérilisation du milieu par enlèvement radical des bancs de vase. Les poissons ne trouvent plus caches ni abris; le cours d'eau ne leur' offre plus guère qu'un milieu de transit (fig. 4).

Viennent s'y ajouter la destruction des zones de fraye :

- des salmonidés, par la suppression des hauts fonds et gravières;

- des esocidés, par la disparition des zones inondées ou des moyens d'y accéder;

- des cyprinidés, par élimination des herbiers.

- Du point de vue de l'environnement et de la pratique de la pêche, on peut noter très fréquemment la destruction du paysage aquatique, une pratique de la pêche rendue difficile par la faible lame d'eau, des amas d'algues, des berges hautes et non stabilisées.

L'ampleur et la durée de ces modifications sont très variables. On estime généralement que la rivière retrouve un aspect acceptable dans un délai de cinq ans.

L'ensemble des perturbations conduit à un basculement des équilibres dans la hiérarchie biologique du milieu 
aquatique. Un bon cours d'eau salmonicole à l'origine peut devenir un mauvais cours d'eau cyprino-ésocicole après un aménagement hydraulique.

Il convient de noter néanmoins certains avantages: enlèvement des vases en excès et des atterrissements, donc un meilleur écoulement des eaux et, dans certains cas, une meilleure alimentation par la nappe phréatique par l'intermédiaire d'un lit non colmaté. Ces mêmes avantages sont d'ailleurs obtenus par un entretien régulier du cours d'eau.

\section{2 - LES ÉCLUSES ET BARRAGES AU FIL DE L'EAU}

Il s'agit des barrages édifiés sur le lit normal des rivières; leur hauteur varie de $1 \mathrm{~m}$ à $3 \mathrm{~m}$. Ils sont souvent destinés à fournir de l'énergie aux usines au fil de l'eau (moulin, micro-centrale, etc.).

Ces retenues sont de faible importance et l'influence du plan d'eau est souvent négligeable. Elles posent toutefois deux problèmes sérieux :

- celui de la manœuvre des ouvrages et de la vidange des biefs ( $(2.1 .1 .2)$;

- celui du franchissement par les migrateurs.

\subsection{1 * Conséquences sur l'aval du cours d'eau}

Les retenues nécessitent d'être vidangées périodiquement pour éviter l'envasement. Les vidanges peuvent toutefois être très préjudiciables aux populations de poissons si elles sont effectuées :

- en période de frai des salmonidés (colmatage des gravières);

— en période chaude (risque de pollution organique).

\subsection{2 - Conséquences sur les migrations}

Les caractéristiques modestes de teis barrages font qu'ils sont rarement équipés de passes à poissons, bien qu'à partir de $1,50 \mathrm{~m}$ une chute soit difficilement franchissable (fig. 5).

Les passes qui existent sont au surplus peu efficaces :

- soit à cause du manque d'entretien;

- soit parce qu'elles ne sont plus suffisamment alimentées en période de migration.

Les faibles débits que l'on rencontre accentuent les difficultés de l'avalaison des smolts; ceux-ci sont aspirés dans les turbines ou entraînés dans des impasses telles que les canaux de prises d'eau.

\section{3 - Les mesures de sauvegarde}

Elles tiennent essentiellement à la prise en considération de tout ce que peut représenter un cours d'eau aux plans de la géographie du paysage, de l'hydraulique, de l'hydrobiologie, de l'économie, mais aussi de l'écologie générale et singulièrement celle du milieu piscicole.

La mobilisation des connaissances relatives à ces notions, et leur exploitation par un aménagement harmonieux, respectant au mieux les intérêts des usagers et ceux du site, peut s'appuyer sur des mesures existantes, souvent judicieuses mais peu connues, peu appliquées ou peut contraignantes.

\section{1 - A L'ÉGARD DES TRAVAUX D'ENTRETIEN}

\subsection{1 - Sur le plan réglementaire}

L'entretien et la manœuvre des ouvrages hydrauliques sont soumis à une réglementation générale, départementale (Arrêtés préfectoraux), locale (Règlements d'eau), individuelle (Cahiers des charges) qu'il conviendrait de mieux faire respecter avec si besoin des peines plus sévères.

La faculté de faire prendre, au niveau départemental, des arrêtés annuels modulant les effets de la réglementation générale en fonction des situations locales et de leur évolution dans le temps, constituerait un premier pas vers la gestion de bassin. Il serait souhaitable en effet qu'un bassin versant, relevant de plusieurs départements, voire de plusieurs régions de programme, puisse jouir d'une personnalité civile, administrative, technique, permettant une gestion hydraulique et piscicole «de tous les jours» harmonieuse et efficace.

La mise au point de conventions annuelles fixant:

- les périodes de faucardement;

- les méthodes de faucardement:

- les périodes de curage;

- les méthodes de curage;

- la localisation des curages;

concevable actuellement pour de petits cours d'eau situés dans un département, et gérés au plan hydraulique par un Syndicat de riverains, pourrait être étendue à de grands cours d'eau souffrant d'un réel écartèlement administratif.

\subsection{2 - Sur le plan technique}

Il conviendrait d'adopter des méthodes étudiées et arrêtées en fonction des caractères hydrauliques, hydrobiologiques et piscicoles du cours d'eau et de l'époque des travaux, par exemple :

- faucarder en layons longitudinaux le milieu des cours d'eau en période du frai des cyprinidés;

- suspendre les curages du $1^{\text {er }}$ novembre au 31 mars dans les zones de gravières favorables à la ponte des salmonidés, ainsi que $5 \mathrm{~km}$ en amont (fig. 6).

\section{2 - A L'ÉGARD DES TRAVAUX NEUFS}

\subsection{1 - Sur le plan réglementaire}

- Obligation de la consultation préalable:

- des services techniques compétents (des Ministères de l'Equipement, de l'Agriculture, de la Qualité de la Vie, de l'Industrie...),

- des collectivités intéressées (Syndicat d'entretien, Fédérations départementales d'A.P.P., A.P.P. locales) dans le cadre de la Circulaire interministérielle du 13 septembre 1974 recommandant une telle concertation.

-- Interdiction de modifier un tracé de cours d'eau, sauf cas exceptionnel mûrement étudié. 

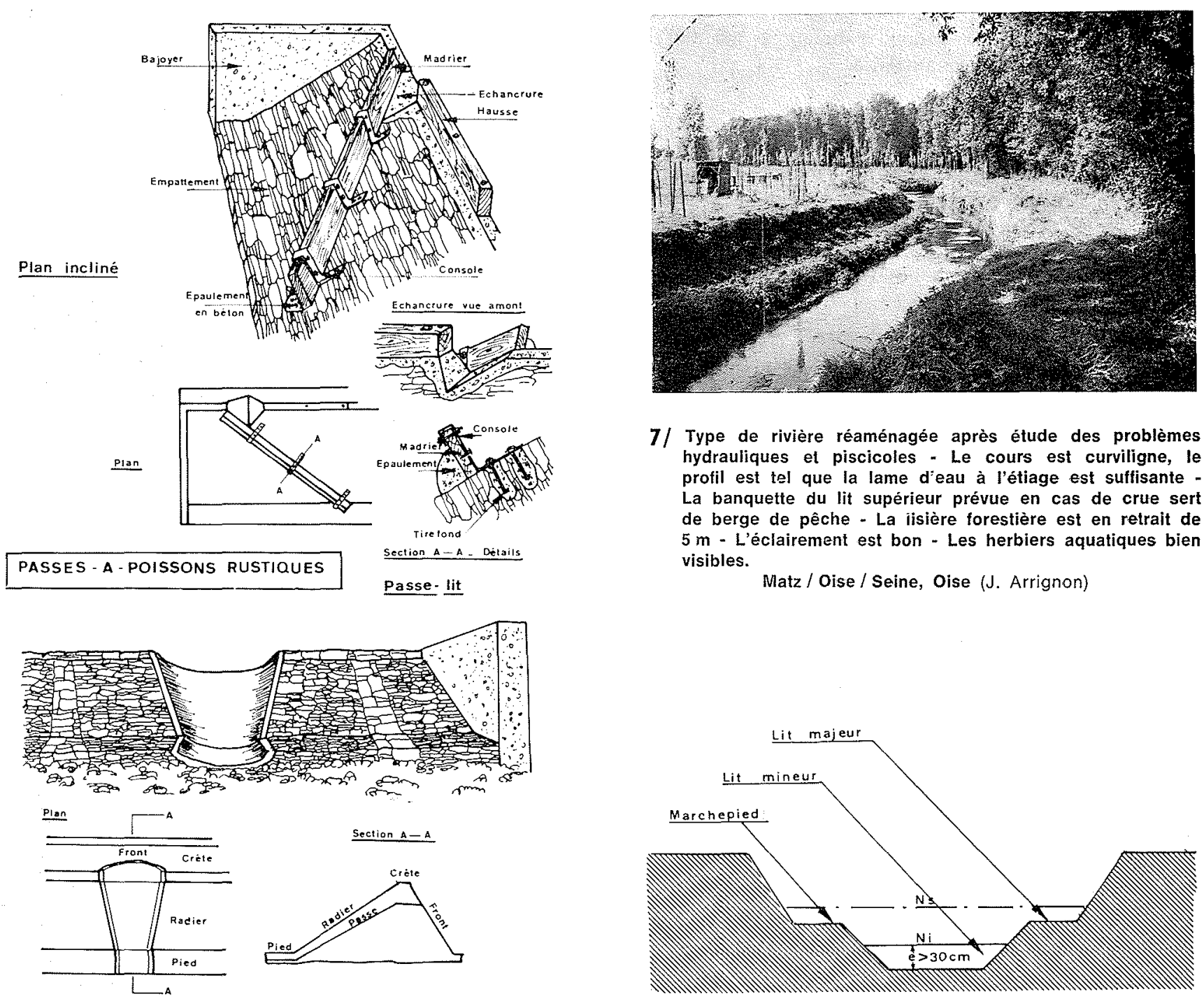

7/ Type de rivière réaménagée après étude des problèmes hydrauliques et piscicoles - Le cours est curviligne, le profil est tel que la lame d'eau à l'étiage est suffisante La banquette du lit supérieur prévue en cas de crue sert de berge de pêche - La iisière forestière est en retrait de $5 \mathrm{~m}$ - L'éclairement est bon - Les herbiers aquatiques bien visibles.

Matz / Oise / Seine, Oise (J. Arrignon)

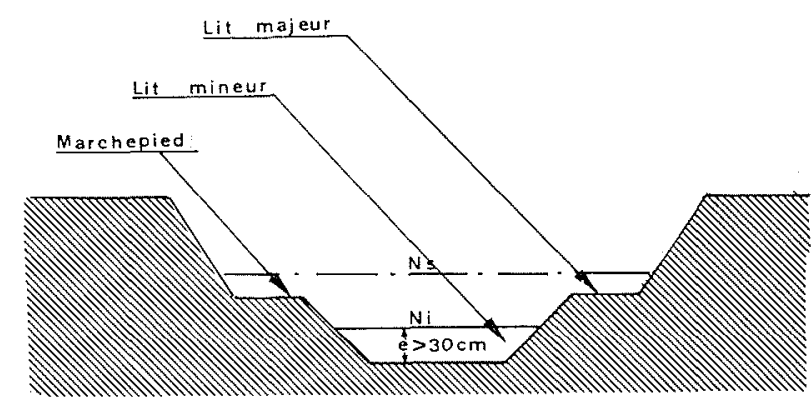

5/ Passes à poissons rustiques (J. Arrignon d'après Brayshaw)

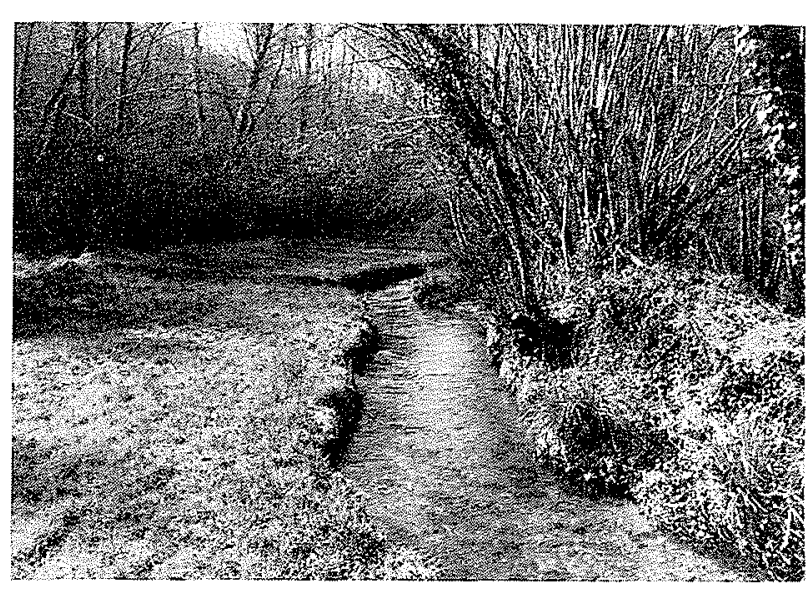

6/ Type de ruisseau bien entretenu - L'éclairement est varié mais bon - Le fond est hétérogène, les caches sont nombreuses.

Affluent du Scorff, Morbihan (J. Arrignon)
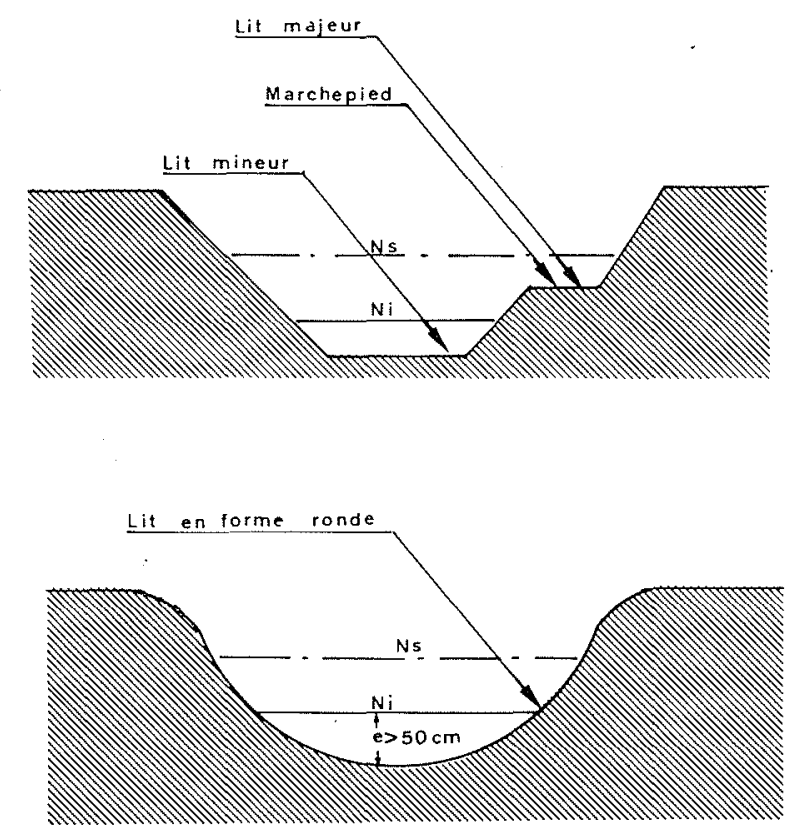

8/ Sections de lit conciliant les impératifs hydrauliques, piscicoles et halieutiques. (J. Arrignon) 
- Octroi de subvention et d'aides financières d'incitation et de réalisation permettant d'aboutir à un objectif piscicole et halieutique amélioré.

\subsection{2 - Sur le plan technique}

- Faire une étude précise justifiant le choix du débit à évacuer : il s'agit de contingences hydrauliques dépendant très certainement plus des moyens disponibles que des méthodes.

- Eviter les tracés rectilignes:

- en conservant le tracé des rivières en équilibre;

- en recoupant éventuellement les méandres accentués, par un bras servant uniquement d'évacuateur de crue.

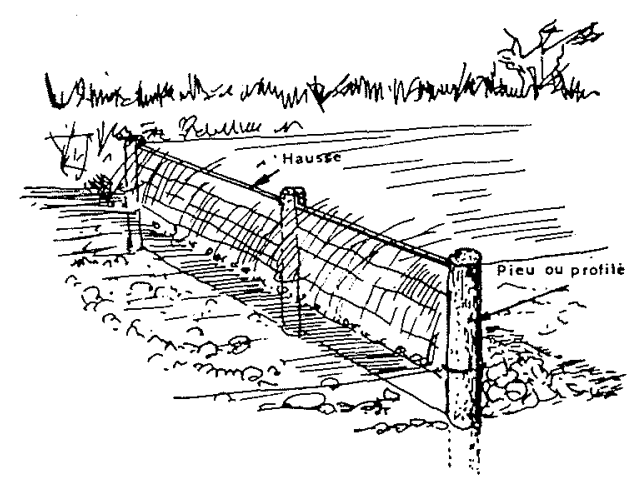

9/ Barrage mobile sur ruisseau de plaine. (J. Arrignon)
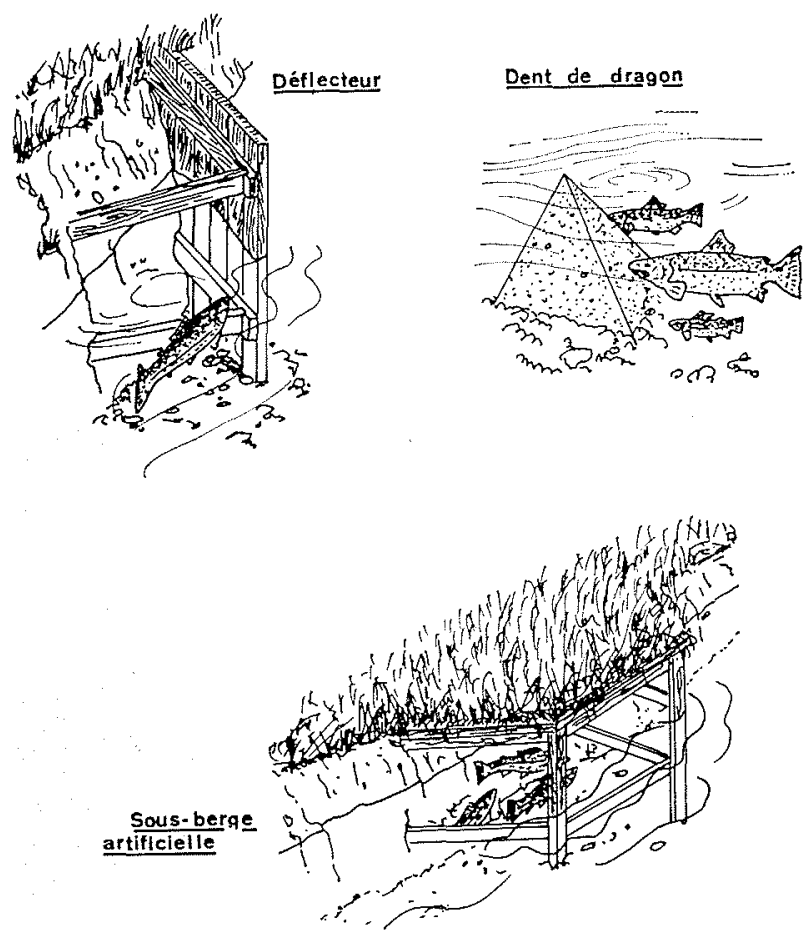

10/ Aménagement de l'habitat du poisson.

$$
\text { (J. Arrignon) }
$$

- Conserver une profondeur d'eau convenable en période d'étiage :

- en aménageant les bras secondaires, quand ils existent, ce qui réduira d'autant l'élargissement du cours principal et le risque d'écoulement en lame mince;

- en créant un chenal dans le lit normal, qui servira de lit mineur (fig. 7 et 8).

- Lors de l'exécution des travaux :

- s'en tenir strictement aux cotes et prescriptions du projet, notamment quand celui-ci prévoit le respect de certains fonds particulièrement biogéniques, le respect d'une rive, le respect d'arbres ou d'arbustes isolés, de cordons de broussailles en bordure (fig. 6).

- Les aménagements complémentaires d'intérêt piscicole consistent en :
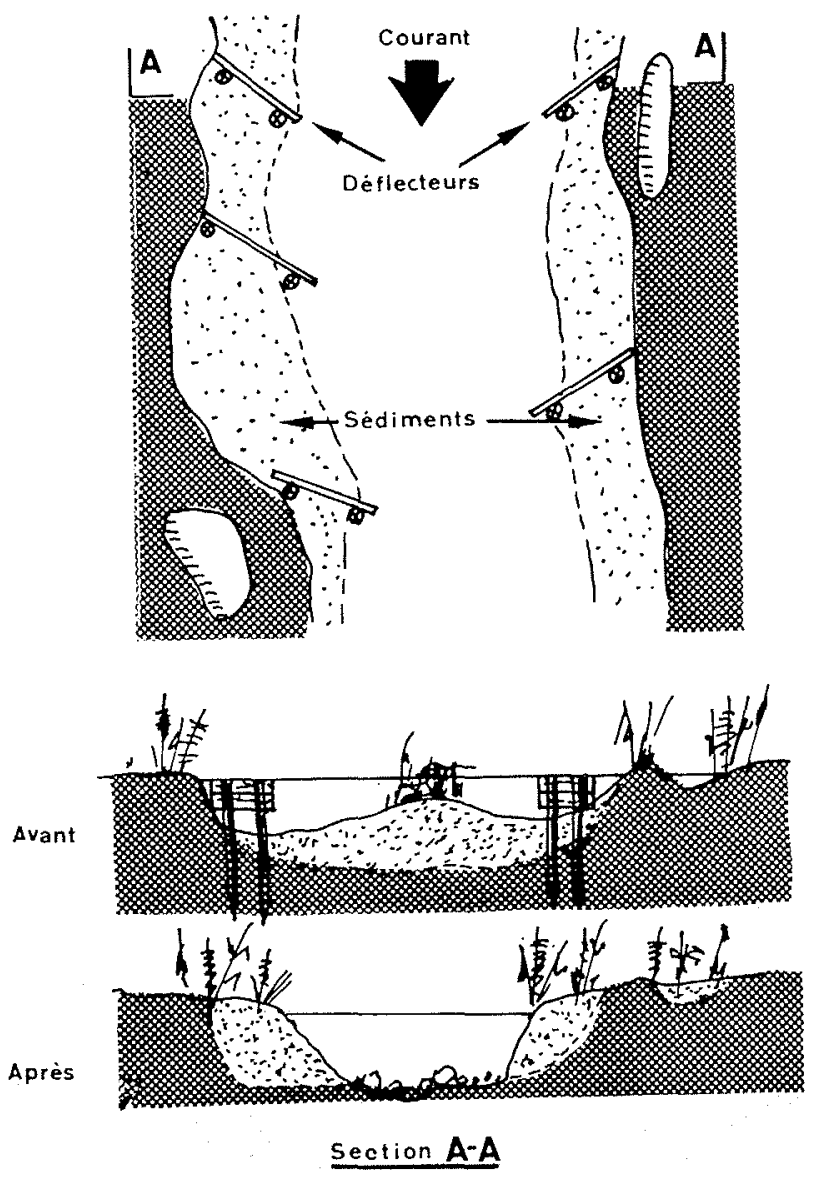

11/ Autocurage.

(J. Arrignon) 
- retenues amovibles, de quelques décimètres de haut, destinées à «casser » le courant et à assurer au poisson une certaine territorialité (fig. 9);

- améliorations de l'habitat du poisson en créant des sous-berges artificielles, des tenues (dents de dragon en quinconce dans le lit), des défiecteurs (fig. 10, 11, 12, 13, 14);

- aménagement de frayères dans les anciens méandres laissés en communication avec la rivière.

- Pour limiter les inconvénients du drainage, il est nécessaire de prendre des mesures anti-érosives (banquettes d'absorption et d'atterrissement) et de prévoir le stockage des eaux d'orage.

\section{4 - Conclusion}

L'accent est porté sur l'aspect écologique et hydrobiologique de l'entretien et de l'aménagement des cours d'eau, plus particulièrement des petits cours d'eau dont l'entretien, notamment, est le plus délaissé et dont, au surplus, le milieu biologique est le plus fragile.

Le relevé des dommages subis par cette partie du réseau hydrographique français, qui en constitue le chevelu, fait ressortir un éventail relativement limité de ces dommages, pouvant être complètement refermé par des mesures de bon sens, par des techniques simples dont la définition assujettie à chaque cours d'eau doit être le fruit de l'imagination de concepteurs pluri-disciplinaires.

Ces interventions dans le milieu doivent en outre être l'effet d'un effort collectif de compréhension, de participation, de vigilance, de «suivi ».

\section{5 - Bibliographie}

Arrignon (J.). - Le problème des fouilles ouvertes en bordure des cours d'eau. La Pisc. Frans., SEDETEC, Paris (1970).

Arrignon (J.). - «Aménagement piscicole des Eaux intérieures》, Ed. SEDETEC, Paris (1970).

Arrignon (J.). - Introduction à l'étude écologique d'un cours d'eau. Rev. Fores. Franc., Nancy (1971).

Arrignon (J.). - «Réaménagement des carrières humides ». Conf. Univers. Orléans (1974). Publ. UNICEM (à paraitre).

Carlier (M.). - "Hydraulique générale et appliquée ». Ed, Eyrolles, Paris (1971).

Dajoz (R.). - «Précis d'Ecologie». Ed. Dunod, Paris (1971).

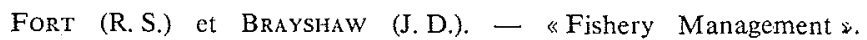
Ed. Faber and Faber, Londres (R.U.) (1961).

Gascorn (R.). - «Retenues collinaires et amónagement rural». Thèse. Ing. Doct. Cons. Nat. des Arts-et-Métiers, Paris (1970).

Mit.s (D.). - «Salmon and trout». Ed. Oliver and Boyd, Edimbourg (R.U.) (1971).

Molinier (R.) et Vignes (P.). - «Ecologie et biocénotique». Ed. Delachaux et Niestlé, Neuchâtel (Suisse) (1971).

Pernes (P.) et Cros (P.). - «Ecoulements à surface libre». Cours d'hydraulique générale de l'ENGREF, Paris.

Porcher (J.-P.) et Porcher-Dechar (C.). - «Etude hydrobiologique et écologique du Bassin de la Bresle ». Mém. DAA-ENSA, Rennes, Doc. du CSP, Paris (1974).

QUESNEL (B.). - «Traité d'hydraulique fluviale et torrentielle. appliquée », tome 2. Ed. Eyrolles, Paris (1973).

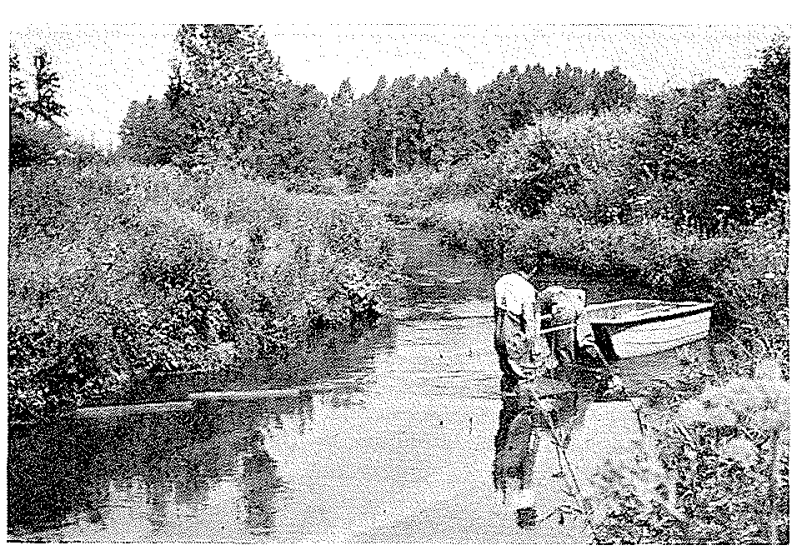

12/ Pose de panneaux déflecteurs pour autocurage d'un petit cours d'eau.

Sainte-Marie / Automne / Oise / Seine, Oise (J. Arrignon)

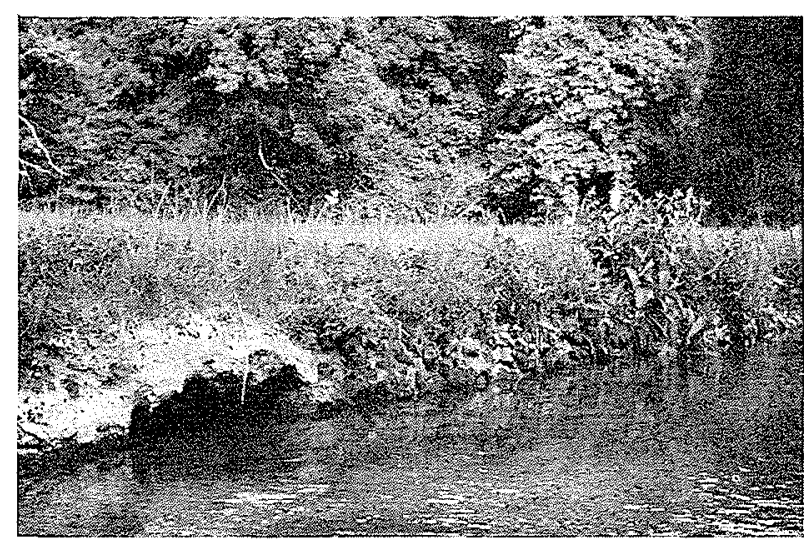

13/ Aménagement de sous-berge artificielle. Risle / Seine, Eure (J. Arrignon)

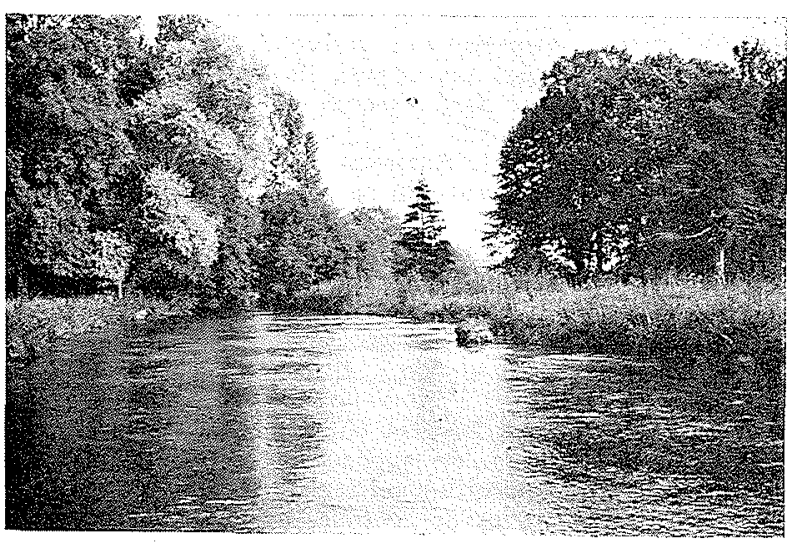

14/ Aménagement d'abris artificiels - Blocs répartis çả et lả dans le lit.

Risle/Seine, Eure (J. Arrignon) 


\section{Discussion}

Président: M. G. LEYNaUd

M. le Président remarcie M. Arrignon pour son exposé bien documenté, très bien illustré et comportant de précieuses indications pratiques. Il ouvre ensuite la discussion.

En Ecosse, dit M. RÉmÉNIÉRAs, la mise en suspension de fines particules organiques résultant de l'exploitation des tourbières et leur dépôt subséquent sur les œufs de salmonidés déposés dans les frayères a entraîné de notables pertes; il ne semble pas que l'on ait pu pallier pratiquement à ces dégâts. Des problèmes analogues se sont-ils posés en France?

En France, répond M. Arrignon, l'exploitation de la tourbe n'est pas importante dans les vallées très riches en truites et en saumons. Mais nous avons un problème analogıe avec les ballastières : c'est un sujet qui fera l'objet d'un certain nombre d'interventions cet après-midi.

On demande, autant que possible, qu'une ballastière sise en bordure d'un petit cours d'eau soit isolée de ce dernier. S'il y a un apport de la ballastière vers le cours d'eau, il se produit une pollution thermique parce que l'eau de celle-ci - surtout si el'e est de grande surface - se réchauffe en été et se refroidit en hiver beaucoup plus que dans le cours d'eau.

Les variations de température $\left(7^{\circ}\right.$ en hiver $; 16$ ou $17^{\circ}$ en été dans le cours d'eau) peuvent s'échelonner entre 22 ol $23^{\circ}$ en été et 2 ou $3^{\circ}$ en hiver dans la ballastière. Les poissons et les invertébrés souffrent énormément d'écarts thermiques a:ıssi importants.

On peut penser atténuer ces écarts en restituant l'eau de fond qui est à $10^{\circ}-11^{\circ}$ comme l'eal de source, mais un tel procédé ne sera pas toujours app'icable en pratique.

M. Receveur (Agence de Bassin Loire-Bretagne) intervient ensuite en ces termes :

Vous avez parlé de «bon sens» dans les moyens à mettre en cuvre et vous avez montré qu'ils étaient assez faciles à réaliser en pratique.

Vous avez parlé aussi d'une réglementation qui oblige, notamment, à une concertation entre les techniciens compétents, les collectivités, l'administration, etc. Or, on consate, et c'est l'impression globale de l'opinion publique, qu'il y a dégradation de l'état des cours d'eau sous les aspects que vous avez évoqués. Alors, je pose les questions suivantes:

- Pour quelles raisons, cette mauvaise situation continue-t-elle, ou s'aggrave-t-elle?

- Est-ce par inapplication de la réglementation ou des possibilités qu'elle donne?

- Est-ce par désintéressement des personnes concernées?

- Quels moyens concrets sont susceptibles de « renverser la vapeur» pour que toutes ces bonnes idées, dont l'intérêt et lat facilité de mise en cuvve sont reconnus far tous dans le principe, ne restent pas un vœu pieux?

Il est certain, répond $M$. Arrignon, qu'il y a une dégradation de la situation et cette dégradation ne date pas de très longtemps : de cinquante ans environ...

Il était dit - et il est toujours dit - dans les textes légaux que le riverain a pour obligation de curer, de faucarder «à vieux fond et vieille berge », le lit du cours d'eau. Or, cela ne se fait plus parce que le riverain, sur les petits cours d'ean, n'a $p^{\top} u s$ les moyens individuels de le faire... ll y a là une négligence qui est un peu une paresse.

En outre, les textes qui faisaient obligation et comportaient certaines contraintes n'ont pas été appliqués pour diverses raisons dont la refonte des services administratifs - depuis 1964 et une carence certaine en moyens de contrôle.

Les contraintes n'ont pas évolué depuis cinquante ans. Je ne sais plus quel est le taux de l'amende en ce qui concerne les prélèvements d'eau abusifs, mais il doit être de l'ordre de 4 francs... C'est là une incitation tout à fait négligeab'e.
Tout le monde parle de pollution et de dégradation; mais fort peu de gens sur le terrain s'en occupent de façon positive. En fait, les habitants d'une vallée ne sont mobilisés que le jour où i's sont atteints dans leurs profits et dans leur manière de vivre.

Je peux citer le cas du Scorff, parce que je le connais bien. C'est un bassin qui est actuellement amélioré, pas seu'ement par les pêcheurs, mais également par les professeurs et les élèves de l'Université de Rennes. Cela provoque de sympathiques sorties d'étudiants venant travailler à l'entretien de la rivière. Finalement, les habitants de la vallée qui, tous, se sentent concernés collaborent aussi aux travaux

Il faudrait que ce genre d'intervention soit étendu à l'ensemble du territoire, car si les cours d'eal sont, dans l'ensemble, mal entretenus, leur dégradaticn ne touche pas, pour le moment, un secteur économique très important.

Les moyens de lutte contre la dégradation des cours d'eau sont, à mon avis:

- l'incitation des intéressés locaux directement concernés;

- une réactualisation de la réglementation en vigueur

- l'étude en commun des objectifs des travaux.

Par exemp'e, lorsqu'un Syndicat intercommunal réunit les moyens de financement nécessaires pour réaménager un cours d'eau, il faudrait qu'au niveau des avant-projets - ou mieux, au niveau des esquisses - les différentes facettes de l'aménagement du cours d'eau soient examinées afin de procéder à l'harmonisation des impératifs à respecter.

Tout ceci correspond à une manière de travailler qui a été bien définie sur le plan interministériel : il existe une circulaire assez éloquente à cet égard : i! suffirait de l'appliquer. D'ailleurs, depuis deux ans, la concertation est beaucoup plus importante et beaucoup plus efficace qu'elle n'était dans le passé.

Pour inciter les populations riveraines à collaborer à l'aménagement de leurs cours d'eau, observe M. RÉMÉniéras, un effort de vulgarisation des techniques élémentaires utilisées à cet effet, serait sans doute bénéfique. Dans un but un pelı analogue, le «Soil Conservation Service » du Ministère de l'Agriculture des EtatsUnis, publie un bulletin mensuel (\%), bien i'lustré, d'une vingtaine de pages, exposant sous une forme simple quelques cas concrets d'aménagement des sols en vue de leur utilisation optimale et de la lutte contre l'érosion. Ce périodique, largement diffusé auprès des élus municipaux, des fermiers, des écoles, etc,, semble avoir suscité une assez large participation des populations intéressées aux travaux locaux de conservation des sols et... de la nature en général.

Bien que beaucoup moins important qu'aux Etats-Unis, le prob'ème de l'érosion des sols, remarque M. le Président, commence à se poser en France sous deux aspects :

Au niveau des rivières, on constate une augmentation généralisée de la turbidité et du transport solide; mais ce problème ne semble pas avoir été encore tellement perçu. M. ArRIGNon y a fait allusion à propos du remodelage du paysage rural.

D'autre part, les nombreux chantiers de Travaux pub'ics qui éventrent un peu partout le sol et souvent sur une grande échelle, grâce aux gros engins de terrassements modernes, sont une cause de dégradation de la qualité des eaux; ils produisent une augmentation de l'érosion qui a des répercussions sur le plan piscicole (co'matage des frayères, disparition de la nourriture pour les poissons, etc.).

M. le Président clôt la discussion et donne la parole à M. VerNEAUX pour l'exposé de sa communication.

(*) Soil Conscrivation. bulletin menstel édité par Government Printing Office, Washington D.C. 20402 


\section{Abstract \\ Protection of fish-farming interests during implementation of river engineering projects}

\section{INTRODUCTION}

The aim of river development is to counteract adverse effects on the river's role as a "conveyor" or "vector". Though river engineering work may also help to protect the aquatic ecology, it also frequently harms the fish population. Appropriate protective measures, therefore, are an important factor to consider in any river development or conservation project.

\section{RIVER ENGINEERING}

River engineering projects with the aim of improving flow conditions include both conservation or maintenance measures and the implementation of new river works.

\section{MAINTENANCE}

Most "private" rivers (i.e. not under State management) genera'ly lack adequate maintenance, being subject to increasing pollution, silting, obstruction and bank degradation (Fig. 1), and also because riparian populations are unab.e to meet their individual obligations.

"Public" (i.e. State-controlled) waterways, on the other hand, are general'y well-maintened, except if down-grated. The latter become eutrophic and obstructed (Fig. 2). In attempting to remedy critical situations arising from a lack of maintenance, inconsiderate action has frequently been taken. e.g. in the form of excessive, ill-timed and ill-placed silt and weed-clearance operations which have caused warping and destroyed various fish species spawning grounds and shetcl spots (Fig. 3).

A total lack of maintenance has very serious consequences, as it results in a kind of "chain-reaction" of physical, chemical and phyto-zoological factors including obstruction, silting, flow reta:dation, rising water temperature and nutrient content, decreasing daylight, destruction of water plants, substitution? of the periphyton and bentos, disorder and upsets in the fish population.

Inconsiderate flow-contro! operations are apt to result in disastrous conditions, e.g. entrainment of toxic sediment, spawning grounds being left high and dry for prolonged periods, etc.

\section{NEW PROJECT IMPLEMENTATION}

Improvement of a river's capacity as a collecting and exchange medium usually involves resizing, profile and bed correction work affecting the physical, chemical and phytozoological conditions and the fish popu'ation's environment. Typical "trouble-factors" include sudden increases in light intensity, temperature and $\mathrm{O}_{2} / \mathrm{CO}_{2}$ variations, and replacement of underwater aquatic regetation by standing vegetation or fibrous water-weed beds, which completely upsets the system in equilibrium within the biological "hierarchy" of the aquatic medium.

Resizing of rivers to standard dimensions tends to du't the diversity of the natural aquatic and "subaquatic" landscape in which fish thrive. Safe hiding places and spawning grounds both become increasingly scarcer.

Silt and deposit clearance does, however, have a number of beneficial effects.

\section{LOCKS AND RIVER BARRAGES}

River barrages are erected on the normal river bed. They have little effect on fish ecology, except in the case of sudden releases of water; which may cause mechanica! or organic pollution. As most barrages are comparatively small, they are seldom equipped with fish-ladders (Fig. 5).

\section{PRotective Measures - MAINTENANCE}

Protective measures under this heading are simply a matter of complying with all water management regu'ations and issuing suitable regulations for such new situations as may arise.

Weed-clearance in strips down the river and silt clearance outside spawning grounds when the fish are not spawning are examples of sensible measures (Fig. 6).

\section{NEW PROJECT IMPLEMENTATION}

Important requirements under this heading include minimal straight river sections (i.e. conservation of bends) and preservation of an adequate depth of water at low-water condition by means of a channel in the normal river bed (Fig. 7 and 8).

On no account should pioject implementation work be allowed to affect the biogenic grounds, specific trees or shrubs or string, of bushes along the river banks (Fig. 6).

Temporary (removable) barrages (Fig. 9) and new fish habitats and spawning grounds (Figs. 10-14) are requirement. serving the fish population which are well worth considering in any river engineering scheme.

\section{CONCLUSION}

Careful'y planned maintenance at regular intervals, sensible aztion and simple techniques "tailored" to suit individual river requirements are vital in preserving the more vulnerable fish species. 